\title{
SÍNTESE E CARACTERIZAÇÃo DE DISPERSÕES AQUOSAS DE POLIURETANOS À BASE DE COPOLÍMEROS EM BLOCO DE POLI(GLICOL ETILÊNICO) E POLI(GLICOL PROPILÊNICO)
}

\author{
Fernanda M. B. Coutinho*, Marcia C. Delpech, Cristiane C. Santos e Rosiléa B. L. Almeida
}

Instituto de Química, Universidade do Estado do Rio de Janeiro, Pavilhão Haroldo Lisboa da Cunha, Rua São Francisco Xavier, 524, 20559-900 Rio de Janeiro - RJ, Brasil

Recebido em 16/9/07; aceito em 14/3/08; publicado na web em 1/9/08

\begin{abstract}
SYNTHESIS AND CHARACTERIZATION OF POLYURETHANE AQUEOUS DISPERSIONS BASED ON POLY(ETHYLENE GLYCOL) AND POLY(PROPYLENE GLYCOL) BLOCK COPOLYMERS. Non-polluting polyurethane aqueous dispersions, with $40 \%$ of solids content, were synthesized based on block copolymers of poly(ethylene glycol) and poly(propylene glycol) (PEG-bPPG), with PEG hydrophilic segments content of 7 and 25\%, poly(propylene glycol) (PPG), dimethylolpropionic acid (DMPA), isophorone diisocyanate (IPDI), and hydrazine. Different formulations were synthesized by varying the equivalent-grams ratios between isocyanate and hydroxyl groups (NCO/OH) and PPG and (PEG-b-PPG). The presence of high amounts of PEG in the formulations provoked the formation of gels. Average particle size and viscosity of the dispersions were determined. Mechanical properties and water absorption resistance of cast films were evaluated.
\end{abstract}

Keywords: polyurethane; aqueous dispersions; non-polluting systems.

\section{INTRODUÇÃO}

A pesquisa e o desenvolvimento de formulações à base de dispersões aquosas poliméricas são de suma importância no que tange à eliminação ou redução de compostos orgânicos voláteis (VOC's) para o meio ambiente, principalmente em aplicações como materiais de revestimento. ${ }^{1}$ Dentre esses materiais, destacam-se as dispersões aquosas poliuretânicas que não são tóxicas, inflamáveis ou poluentes, já que somente a água evapora no processo de secagem. ${ }^{2}$

A produção de poliuretanos (PUR) dispersos em água encontra uma vasta gama de aplicações como revestimentos para uma grande variedade de substratos, entre esses: tecidos, papel, espumas, madeira, couro, vidro, lâminas metálicas e outros substratos poliméricos, e como adesivos. Essa grande versatilidade se deve à ampla variedade de possíveis monômeros que, quando combinados de forma adequada, conferem ao produto final as propriedades desejadas. ${ }^{3-5}$

As principais matérias-primas empregadas na produção dos poliuretanos são os di- ou poliisocianatos e os compostos hidroxilados de baixa ou mesmo alta massa molar (polióis). Dependendo da aplicação do produto final e do tipo de síntese são também usados agentes de cura, agentes de expansão, catalisadores, aditivos, cargas, etc. ${ }^{6}$

Nas cadeias poliuretânicas, duas estruturas básicas podem ser diferenciadas: segmentos longos e moderadamente flexíveis, provenientes dos polióis e segmentos curtos e relativamente rígidos, compreendendo os grupos uretano, derivados dos diisocianatos e dos extensores de cadeia di-hidroxilados, ou grupos uréia, formados a partir de extensores diaminados. As propriedades do polímero formado não dependem somente da razão entre segmentos rígidos e flexíveis, mas também do grau de segregação das fases e da organização tridimensional que possibilitam a formação de estruturas para-cristalinas com fortes interações intermoleculares..$^{711}$

A síntese de poliuretanos é normalmente produzida por poliadição em etapas, basicamente a partir de compostos hidroxilados $(\mathrm{OH})$ e isocianatos (NCO). ${ }^{12,13}$ Os métodos de síntese dos poliuretanos podem ser classificados em função da ordem de adição dos reagentes e do

\footnotetext{
*e-mail: fern@ajato.com.br
}

meio físico no qual ocorre a reação. Dependendo do modo de adição dos reagentes, os processos podem ser classificados em uma ou duas etapas; esse último envolvendo a síntese de um prepolímero. Quanto ao meio reacional, as reações podem ser conduzidas em solução (solventes orgânicos), em dispersão (sistemas aquosos) e em massa (ausência de meio dispersante), sendo esse último o mais utilizado e o mais adequado em termos ambientais. ${ }^{6}$

Na preparação de dispersões, o controle da viscosidade e do tamanho de partícula são parâmetros significativos, principalmente para aplicação específica das dispersões poliuretânicas como revestimentos. ${ }^{14}$

Os poliuretanos, como a maioria dos polímeros sintéticos, não são solúveis em água. ${ }^{15}$ Assim, torna-se necessário promover modificações na estrutura da cadeia poliuretânica de modo a tornar possível a sua dispersão. ${ }^{16}$ Grupos iônicos ou segmentos hidrofílicos, que podem estar presentes nos monômeros iniciais ou no extensor de cadeia, permitem que o polímero se torne compatível com o meio aquoso.,17

Neste trabalho, foram sintetizados poliuretanos aquosos, com hidrofilicidade promovida por grupos aniônicos e segmentos à base de poli(glicol etilênico) (PEG). Foram utilizados, como monômeros, prepolímeros em bloco à base de poli(glicol etilênico) e poli(glicol propilênico) (PEG-b-PPG), com teor de segmentos hidrofílicos (PEG) variando em 7 e 25\%; poli(glicol propilênico) (PPG); ácido dimetilolpropiônico (DMPA); diisocianato de isoforona (IPDI) e hidrazina (HYD), como extensor de cadeia.

\section{PARTE EXPERIMENTAL}

\section{Materiais}

Foram utilizados na síntese das dispersões: ácido dimetilolpropiônico (DMPA), procedência Aldrich Chemical Company, Inc.; copolímeros em bloco à base de poli(glicol etilênico) e poli(glicol propilênico) (PEG-b-PPG), com 7\% e 25\% de PEG, massas molares numéricas médias $\left(\mathrm{M}_{\mathrm{n}}\right)$ iguais a 1850 e $2350 \mathrm{~g} / \mathrm{mol}$, respectivamente, dados informados pelo fabricante, procedência Dow Química S.A; diisocianato de isoforona (IPDI), doação do Centro Técnico Aeroes- 
pacial (CTA); hidrato de hidrazina, solução a 64\% (HYD), doação da Resinac Indústrias Químicas LTDA; poli(glicol propilênico) (PPG), massa molar numérica média $\left(\mathrm{M}_{\mathrm{n}}\right)$ igual a $1300 \mathrm{~g} / \mathrm{mol}$, informada pelo fabricante, procedência Dow Química S.A ; trietilamina (TEA), procedência Vetec Química Fina LTDA.

\section{Síntese}

As sínteses foram conduzidas em duas etapas, em ausência de solvente orgânico. ${ }^{6}$ A primeira etapa consistiu na preparação do prepolímero e neutralização dos grupos carboxílicos provenientes do DMPA. Na segunda etapa, o prepolímero foi disperso em água e, a seguir, foi realizada a reação de extensão de cadeia com hidrazina, dando origem às dispersões poliuretânicas.

Em um reator do tipo kettle, montado sobre uma manta de aquecimento e equipado com agitador mecânico, foram adicionados os polióis (PEG-b-PPG e/ou PPG), o DMPA e o IPDI. A síntese do prepolímero foi conduzida em temperatura na faixa de 90 a $100{ }^{\circ} \mathrm{C}$, durante 15 a 25 min. Em seguida, foi realizada a neutralização com TEA a $40{ }^{\circ} \mathrm{C}$, durante $30 \mathrm{~min}$.

A etapa seguinte foi a dispersão da mistura reacional em água, sob forte agitação. A reação de extensão de cadeia subseqüente foi feita com hidrazina, por $30 \mathrm{~min}$, dando origem a sistemas coloidais binários, nos quais as cadeias poliuretânicas estavam dispersas sob a forma de partículas em uma fase contínua aquosa, como representado na Figura 1.

As dispersões poliuretânicas obtidas foram filtradas em tela de nylon para eliminação de partículas grosseiras. Todas as dispersões obtidas foram preparadas, pelo menos, em duplicata.

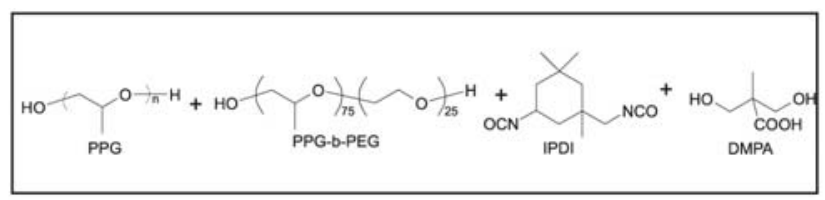

1) Formação do prepolímero

2) $\mathrm{N}(\mathrm{Et})_{3}$

3) Dispersão Aquosa

4) $\mathrm{H}_{2} \mathrm{~N}-\mathrm{NH}_{2}$

\section{Y}

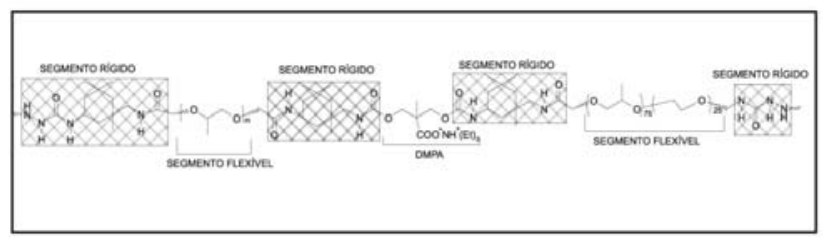

Comportamento da cadeia uretânica

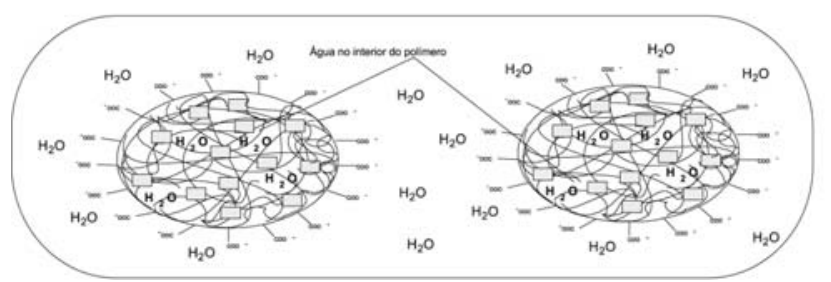

Figura 1. Representação esquemática da síntese dos poliuretanos dispersos em água
Conforme ilustrado na Figura 1, os polióis deram origem aos segmentos flexíveis na cadeia poliuretânica, enquanto os segmentos rígidos, que representam as ligações uretânicas, foram resultantes da reação das hidroxilas terminais dos polióis com os grupos isocianato do IPDI. As ligações ureicas foram formadas na etapa da extensão de cadeia, pela reação da hidrazina com os grupos isocianato terminais presentes no prepolímero.

Nas dispersões aquosas foram variados os seguintes parâmetros: a razão entre o número de equivalentes-grama de grupos isocianato e hidroxila $(\mathrm{NCO} / \mathrm{OH})$ em 1,$7 ; 2,0$ e 2,5 ; a proporção (em equivalentesgrama) de poliol (PPG/PEG-b-PPG) e o teor de PEG presente no copolímero em bloco.

Neste estudo foram introduzidos copolímeros à base de glicol etilênico e glicol propilênico (PEG-b-PPG) com teores de PEG de $7 \%$ e $25 \%$, nomeados séries A e B, respectivamente.

\section{Caracterização}

\section{Dispersões aquosas}

Foram determinados o teor de sólidos ou sólidos totais (ST), o tamanho médio de partículas (TMP), a estabilidade frente à sedimentação e a viscosidade em fluxo contínuo das dispersões aquosas poliuretânicas.

O teor de sólidos ou sólidos totais corresponde à porção nãovolátil presente nas dispersões. Foram sintetizadas dispersões aquosas com teor de sólidos totais teórico de $40 \%$. Os valores experimentais foram determinados segundo método padrão. ${ }^{18}$ Esse teor é um parâmetro relevante na avaliação de perdas eventuais durante a síntese do poliuretano, na etapa da dispersão do prepolímero e está associado à viscosidade do mesmo.

O tamanho médio de partícula (TMP) foi determinado por espalhamento de luz (LS) em analisadores Malvern Mastersizer 2000 e Zetasizer Nano, que permitem detecções de tamanho de partícula na escala de 0,02 a $2000 \mu \mathrm{m}$ e $0,6 \mathrm{~nm}$ a $6 \mu \mathrm{m}$, respectivamente. Foram adotados dois equipamentos devido às diferentes características das formulações obtidas. Poliuretanos preparados a partir do copolímero com teor de $25 \%$ de PEG (Série B) produziram dispersões altamente viscosas e com tamanho médio de partícula elevado, necessitando de maior faixa de detecção que os sistemas obtidos a partir do copolímero com $7 \%$ de teor de PEG (Série A). Nesse último caso, o nível de detecção teve que apresentar maior sensibilidade.

A análise viscosimétrica das dispersões aquosas foi feita a 25,0 $\pm 0,1^{\circ} \mathrm{C}$ em um viscosímetro digital Brookfield, modelo LV DVII + Pro, utilizando dois diferentes rotores (spindles), SCA-31 e SCA-18 (a seleção de cada spindle foi de acordo com a faixa específica da viscosidade das dispersões) empregando-se um adaptador UL universal para volumes reduzidos de amostra, acoplado a banho termostatizado Haake. O equipamento foi calibrado com padrão de viscosidade conhecida conforme instrução do fabricante. A velocidade (ou taxa de cisalhamento) não pôde ser fixada porque as formulações estudadas apresentaram viscosidades diferentes. Foi necessária a realização de ajustes da velocidade para se conseguir a leitura dentro da escala, ou dentro dos limites de confiabilidade.

\section{Filmes vazados}

Os filmes de poliuretanos foram obtidos por vazamento das dispersões em superfícies niveladas de Teflon e secas ao ar por um período de 7 dias. As características de formação dos filmes foram avaliadas visualmente. Depois de desmoldados, os filmes foram caracterizados por espectroscopia na região do infravermelho (FTIR) e suas propriedades mecânicas e resistência à água foram avaliadas.

A adesão dos revestimentos obtidos a partir da aplicação das dispersões foi avaliada em superfícies de Teflon, vidro, alumínio, 
madeira, papel e cerâmica. As dispersões foram vazadas e espalhadas sobre essas superfícies, de modo a formar uma fina película após secagem por 1 semana à temperatura ambiente. ${ }^{19}$

As análises de FTIR foram conduzidas em espectrômetro Perkin Elmer Spectrum One, pelo método de refletância total atenuada (ATR), com resolução de $4 \mathrm{~cm}^{-1}$ e com 32 varreduras.

O percentual de absorção de água dos filmes foi determinado em duplicata por método padrão, ${ }^{20}$ durante 20 dias. A avaliação das propriedades mecânicas foi feita em dinamômetro Instron 5565, com célula de carga de $100 \mathrm{~N}$ e velocidade de separação das garras de $500 \mathrm{~mm} / \mathrm{min}$, própria para borrachas, visto que os filmes vazados apresentaram propriedades elastoméricas. Os corpos de prova apresentaram dimensões 5 x $70 \mathrm{~mm}$, de acordo com a adaptação de método padrão. ${ }^{21}$

\section{RESULTADOS E DISCUSSÃO}

\section{Tamanho de partícula e teor de sólidos das dispersões aquosas}

Uma partícula de poliuretano dispersa é formada por uma camada interna, composta de porções hidrofóbicas, e uma camada externa composta de porções hidrofílicas, que podem ser constituídas de segmentos hidrofílicos, contendo ou não grupos iônicos, e também de ligações uretânicas e ureicas. ${ }^{4,22}$

Nas partículas provenientes das dispersões analisadas, pode-se supor que a camada externa é formada pelos grupos carboxilato provenientes do DMPA, pelas ligações uretânicas e ureicas e pelos segmentos PEG presentes no copolímero em bloco. A camada interna, hidrofóbica, é formada pelos segmentos hidrofóbicos do PPG e dos blocos de PPG presentes no copolímero.

O tamanho médio das partículas, em dispersões poliuretânicas, pode variar entre 10 a $5000 \mathrm{~nm}$ e define a estabilidade da dispersão frente à sedimentação. Partículas maiores que 1000 nm, em geral, são instáveis em relação à sedimentação, enquanto partículas com diâmetros menores ou em torno de $200 \mathrm{~nm}$ produzem dispersões estáveis o suficiente para serem estocadas. ${ }^{23,24}$
O teor de sólidos, de forma geral, está relacionado à viscosidade do prepolímero e ao tamanho médio de partícula das dispersões. Quanto maior for a viscosidade do prepolímero, maior será a sua dificuldade em se dispersar no meio reacional, o que pode provocar a formação de partículas grosseiras que não serão incorporadas à dispersão, levando a uma perda de sólidos no final da reação. Esse aumento na viscosidade pode ser devido a fatores como aumento no teor de poliol, com conseqüente redução na razão $\mathrm{NCO} / \mathrm{OH}$, por exemplo, que pode levar a um aumento na massa molar do prepolímero. ${ }^{25}$

As Tabelas 1 e 2 mostram os valores do teor de sólidos e do tamanho médio de partícula das dispersões das séries A e B produzidas.

Pode-se observar, em ambas as tabelas, que o teor médio de sólidos totais das dispersões das séries A e B produzidas ficou em torno de $37 \pm 2 \%$. Tendo em vista que os cálculos foram feitos para a obtenção de dispersões aquosas com teor de sólidos de $40 \%$, podese concluir que o rendimento das reações foi satisfatório, com baixa perda de massa, tanto na etapa da dispersão do prepolímero em água, quanto frente à sedimentação das partículas já dispersas.

De forma geral, o aumento da quantidade de copolímero na formulação, especialmente na razão $\mathrm{NCO} / \mathrm{OH}$ mais baixa, provocou tanto um aumento na viscosidade do polímero, quanto na hidrofilicidade das cadeias poliméricas, o que levou à formação de partículas maiores. Altas viscosidades dificultaram a dispersão do prepolímero e a hidrofilicidade promovida pelos segmentos PEG provocou inchamento das partículas e agregação com sedimentação, o que, consequientemente, levou a uma diminuição do teor de sólidos.

O tamanho médio de partícula das dispersões da série A (Tabela 1) variou entre 95 e $228 \mathrm{~nm}$. Esses valores ficaram praticamente todos dentro da faixa em que as dispersões são consideradas estáveis. ${ }^{2} \mathrm{O}$ tamanho médio de partícula das dispersões instáveis foi medido após sedimentação das partículas maiores. A análise foi realizada com o sobrenadante e, por isso, os resultados não seguiram um padrão de comportamento.

Os dados obtidos para o tamanho médio de partícula indicaram que a presença de segmentos PEG, em maiores proporções prejudicou a formação de dispersões estáveis. O alto caráter hidrofílico dos seg-

Tabela 1. Teor de sólidos (ST\%) e tamanho médio de partículas (TMP) das dispersões da série A (copolímero com 7\% EG)

\begin{tabular}{|c|c|c|c|c|c|c|}
\hline \multirow{2}{*}{$\%$ EG-b-PG ${ }^{A}$} & \multicolumn{2}{|c|}{$\mathrm{NCO} / \mathrm{OH}=1,7$} & \multicolumn{2}{|c|}{$\mathrm{NCO} / \mathrm{OH}=2,0$} & \multicolumn{2}{|c|}{$\mathrm{NCO} / \mathrm{OH}=2,5$} \\
\hline & TMP (nm) & ST $(\%)$ & TMP (nm) & ST $(\%)$ & TMP (nm) & $\mathrm{ST}(\%)$ \\
\hline 0 & 95 & 38 & 137 & 37 & ND & 39 \\
\hline 30 & 142 & 38 & 181 & 37 & 112 & 37 \\
\hline 50 & 116 & 35 & 212 & 38 & 133 & 39 \\
\hline 70 & $45^{*}$ & 35 & $228 *$ & 36 & ND & 38 \\
\hline 100 & $183^{*}$ & 35 & $201 *$ & 38 & ND & 38 \\
\hline
\end{tabular}

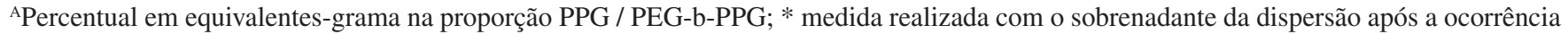
da sedimentação; ND - não determinado

Tabela 2. Teor de sólidos (ST\%) e tamanho médio de partículas (TMP) das dispersões da série B (copolímero com 25 \% EG)

\begin{tabular}{|c|c|c|c|c|c|c|}
\hline \multirow{2}{*}{$\%$ EG-b-PG ${ }^{\mathrm{A}}$} & \multicolumn{2}{|c|}{$\mathrm{NCO} / \mathrm{OH}=1,7$} & \multicolumn{2}{|c|}{$\mathrm{NCO} / \mathrm{OH}=2,0$} & \multicolumn{2}{|c|}{$\mathrm{NCO} / \mathrm{OH}=2,5$} \\
\hline & TMP (nm) & $\mathrm{ST}(\%)$ & TMP (nm) & ST $(\%)$ & TMP (nm) & ST $(\%)$ \\
\hline 0 & ND & 38 & 391 & 36 & 399 & 36 \\
\hline 5 & 173 & 36 & - & - & - & - \\
\hline 10 & $1531 *$ & $30 *$ & 395 & 37 & 572 & 39 \\
\hline 15 & $8623 *$ & $26^{*}$ & - & - & - & - \\
\hline 20 & 23252 & 37 & $3208^{*}$ & 37 & 6480 & 38 \\
\hline 30 & - & - & $2682 *$ & 37 & 12587 & 39 \\
\hline
\end{tabular}

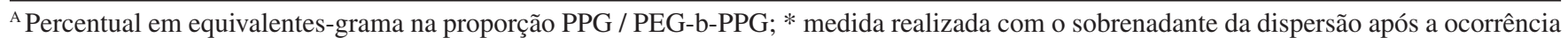
da sedimentação; ND - não determinado 
mentos de glicol etilênico aumentou tanto a afinidade das dispersões pela água, que prejudicou a formação das partículas e aumentou a viscosidade do meio, formando um sistema agregado com aspecto de gel, descaracterizando, dessa forma, a dispersão. Quanto maior o teor de segmentos PEG, maior foi a hidrofilicidade e maior a tendência à formação de gel, o que pode ser observado, principalmente, nas razões NCO/OH mais baixas. Dessa forma, verificou-se que não é possível a obtenção de dispersões, na série B (copolímero com 25\% de segmentos PEG), utilizando teores de copolímero acima de $30 \%$, em relação à razão $\mathrm{PPG} / \mathrm{PEG}-\mathrm{b}-\mathrm{PPG}$, para qualquer uma das razões $\mathrm{NCO} / \mathrm{OH}$ testadas.

Dois fatores, individual ou concomitantemente, relacionados ao enrijecimento das cadeias, podem também ter contribuído para o aumento do tamanho das partículas. Ambos estariam relacionados ao melhor empacotamento das mesmas, com elevação no teor de cristalinidade, e consequiente aumento na rigidez, o que provocaria uma dificuldade maior na formação das partículas. Um deles seria a presença dos segmentos PEG, com características cristalinas, em oposição aos segmentos PPG, que apresentam grupos metila pendentes. $\mathrm{O}$ outro fator seria a presença dos segmentos rígidos, fortemente associados por ligações de hidrogênio, formados pelos grupos uretânicos e ureicos.

\section{Estabilidade das dispersões frente à sedimentação}

Segundo a literatura, a estabilidade frente à sedimentação das dispersões aquosas de $\mathrm{PU}$ cresce à medida que a razão $\mathrm{NCO} / \mathrm{OH}$ aumenta. ${ }^{25}$ Nesse contexto, ao se manter fixa a proporção de (PPG/ PEG-b-PPG) na série A e B foi observado experimentalmente que, dentre as razões $\mathrm{NCO} / \mathrm{OH}$ utilizadas $(1,7 ; 2,0$ e 2,5), a razão $\mathrm{NCO} /$ $\mathrm{OH}=1,7$ apresentou dispersões menos estáveis. Em contrapartida, em grande parte das razões $\mathrm{NCO} / \mathrm{OH}=2,0$ e 2,5 foram produzidas dispersões estáveis (ausência de sedimentação); levando-se em conta o período de até 6 meses após a síntese desse material.

Pode-se supor que o aumento da razão $\mathrm{NCO} / \mathrm{OH}$ facilitou a dispersão pois, aparentemente, o diisocianato atuou como um diluente no meio reacional na síntese do prepolímero e, ao mesmo tempo, a proporção de poliol era menor. Além disso, a maior estabilidade das dispersões também pode ser associada ao aumento do número de ligações de hidrogênio com o aumento da razão $\mathrm{NCO} / \mathrm{OH}$ que, conseqüentemente, facilita as interações do grupo N-H com o oxigênio da água.

A proporção de PPG/PEG-b-PPG esteve também diretamente associada à estabilidade das dispersões. O efeito de PPG/PEG-bPPG foi acentuadamente diferenciado para as dispersões com teores de PEG de $7 \%$ e de $25 \%$. Considerando as dispersões da série A, o aumento no teor em equivalentes-grama de copolímero na proporção PPG/PEG-b-PPG não dificultou de forma significativa a dispersão do prepolímero. Entretanto, influenciou a estabilidade das dispersões, quanto à sedimentação. Com razões $\mathrm{NCO} / \mathrm{OH}$ iguais a 1,7 e 2,0 e proporção 30 PPG/70 PEG-b-PPG, as dispersões produzidas não se mantiveram estáveis, apresentando depósito em forma de gel, após 15 dias da síntese. Já, com a incorporação de $100 \%$ de copolímero, o caráter cristalino desses segmentos pode ter sobressaído, provocando um melhor empacotamento entre esses segmentos de cadeia, competindo com a hidrofilicidade e resultando, assim, em uma dispersão com maior estabilidade mesmo 15 dias após a síntese.

Na série B, o emprego de $10 \%$ de copolímero e razão $\mathrm{NCO} / \mathrm{OH}$ $=2,0$ produziu uma dispersão estável, mas quando se empregou 20 e 30\% do copolímero ocorreu segregação entre as fases após aproximadamente 15 dias. Para a razão $\mathrm{NCO} / \mathrm{OH}=2,5$, as dispersões se apresentaram homogêneas, sem formação de gel, até a proporção de $10 \%$ de copolímero. Entretanto, nas proporções de 20 e $30 \%$ de copolímero, foram gerados materiais altamente viscosos e com características adesivas em substratos como porcelana e madeira.

\section{Viscosidade em fluxo contínuo}

Devido à ampla faixa de viscosidade observada nas dispersões, foi necessário o uso de dois rotores (spindles) para a determinação da viscosidade em fluxo contínuo. Mesmo assim, algumas dispersões não puderam ser avaliadas no viscosímetro Brookfield, pois apresentaram viscosidade fora da escala de medidas possíveis nesse experimento.

As dispersões aquosas analisadas, das séries A e B apresentaram, em geral, característica de fluidos pseudoplásticos, ${ }^{26}$ ou seja, a viscosidade das dispersões diminuiu com o aumento da taxa de cisalhamento como ilustrado na Figura 2.

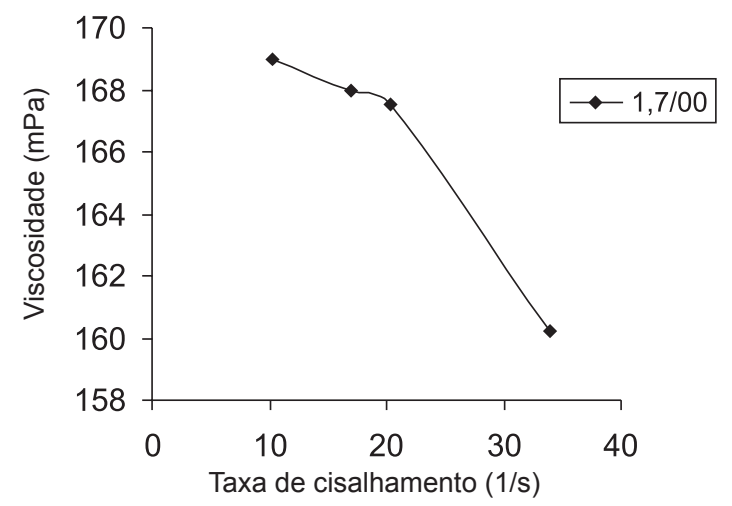

Figura 2. Curva de viscosidade versus taxa de cisalhamento para a série $B$, na razão $\mathrm{NCO} / \mathrm{OH}=1,7$ e com $0 \%$ de $P P G-b-P E G$

As Tabelas 3 e 4 mostram os resultados obtidos para as séries $\mathrm{A}$ e $\mathrm{B}$, respectivamente, fixando-se a razão $\mathrm{NCO} / \mathrm{OH}=2,5$. Pode-se observar que, na série A, na qual o copolímero apresenta menor teor de PEG, a viscosidade teve um aumento pouco pronunciado com o aumento na proporção de copolímero na formulação. Já as dispersões da série $\mathrm{B}$, nas quais o copolímero apresentou maior teor de segmentos PEG, apresentaram um aumento significativo nos valores de viscosidade em função do aumento do teor de copolímero.

De forma geral, pode-se notar que, em todas as razões $\mathrm{NCO} / \mathrm{OH}$ testadas, o aumento do teor de copolímero em bloco, com conseqüente aumento no tamanho de partícula, resultou, portanto, em um aumento na viscosidade da dispersão.

A Tabela 5 mostra a variação dos valores de viscosidade observada na série B para a proporção fixa de $10 \%$ de copolímero com variação na razão $\mathrm{NCO} / \mathrm{OH}$. Pode-se verificar que a viscosidade diminuiu acentuadamente com o aumento da razão $\mathrm{NCO} / \mathrm{OH} .{ }^{12} \mathrm{O}$ aumento nessa última indica uma menor quantidade de poliol na formulação.

\section{Características adesivas}

A adesão foi qualitativamente avaliada por aplicação da dispersão aquosa em superfícies de Teflon, alumínio, vidro, madeira, papel e cerâmica. Verificou-se que quase todas as dispersões produzidas aderiram às superfícies testadas. Não ocorreu adesão somente nas dispersões vazadas em superfícies de Teflon, permitindo assim que os filmes fossem analisados mecanicamente após desmoldagem dessa superfície.

A não-aderência dos filmes de poliuretano às superfícies de Teflon pode ser atribuída à grande diferença de polaridade existente entre os filmes (polares) e o substrato (apolar). No entanto, a aderência dos filmes ao vidro (grupos silanóis), à madeira e ao papel (celulose) 
Tabela 3. Viscosidade em função da taxa de cisalhamento da dispersão da série A na razão $\mathrm{NCO} / \mathrm{OH}=2,5$

\begin{tabular}{lccccccc}
\hline 0\% PEG-b-PPG & \multicolumn{2}{c}{$30 \%$ PEG-b-PPG } & \multicolumn{2}{c}{$50 \%$ PEG-b-PPG } & \multicolumn{2}{c}{$70 \%$ PEG-b-PPG } \\
\hline$\eta(\mathrm{mPa})$ & $\gamma\left(\mathrm{s}^{-1}\right)$ & $\eta(\mathrm{mPa})$ & $\gamma\left(\mathrm{s}^{-1}\right)$ & $\eta(\mathrm{mPa})$ & $\gamma\left(\mathrm{s}^{-1}\right)$ & $\eta(\mathrm{mPa})$ & $\gamma\left(\mathrm{s}^{-1}\right)$ \\
40 & 18 & 40 & 24 & 40 & 25 & 40 & 33 \\
66 & 18 & 66 & 24 & 66 & 24 & 66 & 31 \\
79 & 17 & 79 & 23 & 79 & 23 & 79 & 30 \\
132 & 17 & 132 & 22 & 132 & 23 & 132 & 27 \\
\hline
\end{tabular}

$\eta$ - Viscosidade determinada em viscosímetro Brookfield, $\gamma$ - taxa de cisalhamento

Tabela 4. Viscosidade em função da taxa de cisalhamento da dispersão da série B na razão NCO/OH=2,5

\begin{tabular}{lccccccc}
\hline 0\% PEG-b-PPG & & \multicolumn{2}{c}{$10 \%$ PEG-b-PPG } & \multicolumn{2}{c}{$20 \%$ PEG-b-PPG } & \multicolumn{2}{c}{$30 \%$ PEG-b-PPG } \\
\hline$\eta(\mathrm{mPa})$ & $\gamma\left(\mathrm{s}^{-1}\right)$ & $\eta(\mathrm{mPa})$ & $\gamma\left(\mathrm{s}^{-1}\right)$ & $\eta(\mathrm{mPa})$ & $\gamma\left(\mathrm{s}^{-1}\right)$ & $\eta(\mathrm{mPa})$ & $\gamma\left(\mathrm{s}^{-1}\right)$ \\
5 & 132 & 18 & 40 & 3079 & 0,5 & 13557 & 0,2 \\
- & - & 18 & 66 & 3164 & 0,7 & 13647 & 0,2 \\
- & - & 17 & 79 & 3179 & 0,8 & 13407 & 0,3 \\
- & - & 16 & 132 & 3169 & 1 & 12957 & 0,5 \\
- & - & - & - & 3134 & 1 & 12537 & 0,7 \\
- & - & - & - & 3071 & 2 & - & - \\
- & - & - & - & 3009 & 2 & - & - \\
- & - & - & - & 2798 & 3 & & - \\
\hline
\end{tabular}

$\eta$ - Viscosidade determinada em viscosímetro Brookfield, $\gamma$ - taxa de cisalhamento

Tabela 5. Viscosidade em função da taxa de cisalhamento da dispersão da série B com $10 \%$ dePEG-b-PPG e variação na razão $\mathrm{NCO} / \mathrm{OH}$

\begin{tabular}{lccccc}
\hline Razão $\mathrm{NCO} / \mathrm{OH}=$ & \multicolumn{2}{c}{ Razão $\mathrm{NCO} / \mathrm{OH}=$} & \multicolumn{2}{c}{ Razão $\mathrm{NCO} / \mathrm{OH}=$} \\
1,7 & \multicolumn{2}{c}{2,0} & \multicolumn{2}{c}{} \\
\hline$\eta(\mathrm{mPa})$ & $\gamma\left(\mathrm{s}^{-1}\right)$ & $\eta(\mathrm{mPa})$ & $\gamma\left(\mathrm{s}^{-1}\right)$ & $\eta(\mathrm{mPa})$ & $\gamma\left(\mathrm{s}^{-1}\right)$ \\
78 & 8 & 24 & 0,6 & 18 & 40 \\
78 & 13 & 24 & 1 & 18 & 66 \\
78 & 16 & 23 & 1 & 17 & 79 \\
77 & 26 & 23 & 2 & 16 & 132 \\
77 & 40 & 22 & 3 & $\mathrm{ND}$ & $\mathrm{ND}$ \\
\hline
\end{tabular}

$\eta$ - Viscosidade em fluxo contínuo; $\gamma$ - taxa de cisalhamento; ND não determinada

e à cerâmica (sílica a e argila) pode ser atribuída à alta polaridade desses substratos.

A aderência dos filmes de poliuretano ao substrato de alumínio pode ser resultante do fato dos átomos de nitrogênio e de oxigênio dos PUR possuírem um par de elétrons livres (base de Lewis) que interage com os orbitais vazios da superfície metálica, boa receptora de elétrons.

\section{Caracterização dos filmes vazados por espectroscopia de infravermelho}

$\mathrm{Na}$ análise de poliuretanos por espectroscopia na região do infravermelho (FTIR), um dado importante é o desaparecimento da banda de NCO livre (em torno de $2265 \mathrm{~cm}^{-1}$ ) e o aparecimento da banda em $3300 \mathrm{~cm}^{-1}$ (estiramento $\mathrm{NH}$ ). Essas bandas indicam que a reação foi completa, e um poliuretano foi formado. ${ }^{15,23}$ Em princípio, a presença de grupos uretânicos é verificada pelo aparecimento das bandas em torno de $1538 \mathrm{~cm}^{-1}$ (amida II), $1724 \mathrm{~cm}^{-1}$ (amida I) e $3330 \mathrm{~cm}^{-1}$ (N-H). ${ }^{27,28}$

A carbonila uretânica absorve na faixa de 1696 a $1702 \mathrm{~cm}^{-1}$ e a carbonila ureica pode corresponder a freqüências mais baixas, na faixa de 1636 a $1684 \mathrm{~cm}^{-1}$. Além disso, vale ressaltar que carbonilas ureicas associadas por ligações de hidrogênio podem ser relacionadas a absorções na faixa de 1640 a $1660 \mathrm{~cm}^{-1}$; enquanto que carbonilas ureicas não-associadas por ligação de hidrogênio podem ser relacionadas a absorções em $1695 \mathrm{~cm}^{-1}$. 29,30

A incorporação do copolímero em bloco (PEG-b-PPG) provocou deslocamentos pouco significativos nos espectros de absorção correspondentes aos grupos $\mathrm{N}-\mathrm{H}$ (uretânico e ureico) e $\mathrm{C}=\mathrm{O}$ (uretânico) e alterou pouco as suas intensidades, independente da razão $\mathrm{NCO} /$ $\mathrm{OH}$ empregada. Os espectros de ambas as séries estudadas exibiram perfis similares. A Figura 3 mostra os espectros de absorção dos filmes obtidos em razão $\mathrm{NCO} / \mathrm{OH}=1,7$ para a série $\mathrm{B}$.

A Figura 4 representa espectros da série A de filmes de PUR nas três razões NCO/OH estudadas, com teor fixo de 70\% de PEG-b-PPG. De acordo com o espectro, pode-se notar que há um desdobramento da banda na região da carbonila, verificado também para a série B. Para a razão NCO/OH mais baixa, a banda correspondente às ligações uretânicas, em números de ondas mais elevados, é mais expressiva em comparação à absorção relativa às ligações uréia, que aparece como um ombro. Convém ressaltar que maiores razões $\mathrm{NCO} / \mathrm{OH}$ requerem maiores quantidades de extensor de cadeia na reação sendo maior o teor de ligações ureicas formadas. Dessa forma, com o aumento da razão $\mathrm{NCO} / \mathrm{OH}$, a intensidade do desdobramento relativo às ligações ureicas fica mais expressivo.

\section{Absorção de água dos filmes de PUR}

A baixa resistência à água dos PUR é uma característica dessa classe de materiai ${ }^{31}$ que pode não ser benéfica em determinadas aplicações. No entanto, isso pode ser uma vantagem, principalmente, quando aliada à alta afinidade pela água do segmento de poli(glicol etilênico), o que pode tornar os PUR aplicáveis como materiais superabsorventes. ${ }^{32}$

Kim e $\mathrm{Lee}^{33}$ ao produzirem poliuretanos à base de IPDI dispersos em meio aquoso e diferentes polióis, poli(tetrametileno glicol) (PTMG), poli(adipato de tetrametileno) (PTAd), PPG e PEG, observaram que a substituição de $20 \%$ de PTAd por PEG na formulação fez o inchamento dos filmes em água aumentar de 50 para $220 \%$ em apenas $24 \mathrm{~h}$. Os autores recomendaram que o PEG só deveria ser utilizado nas formulações de poliuretanos dispersos em água na forma de copolímero devido à sua alta sensibilidade à água. 


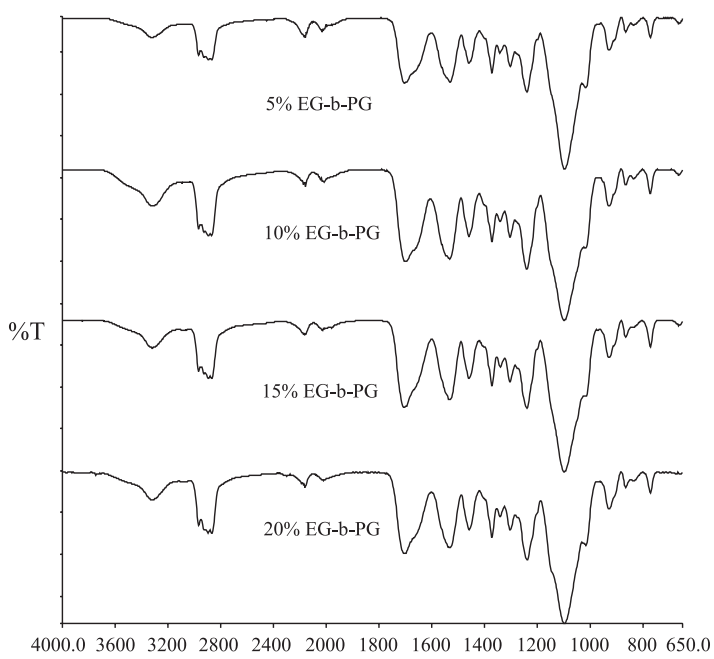

$\mathrm{cm}^{-1}$

Figura 3. Espectros de absorção dos filmes de PUR da série B na razão $\mathrm{NCO} / \mathrm{OH}=1,7$

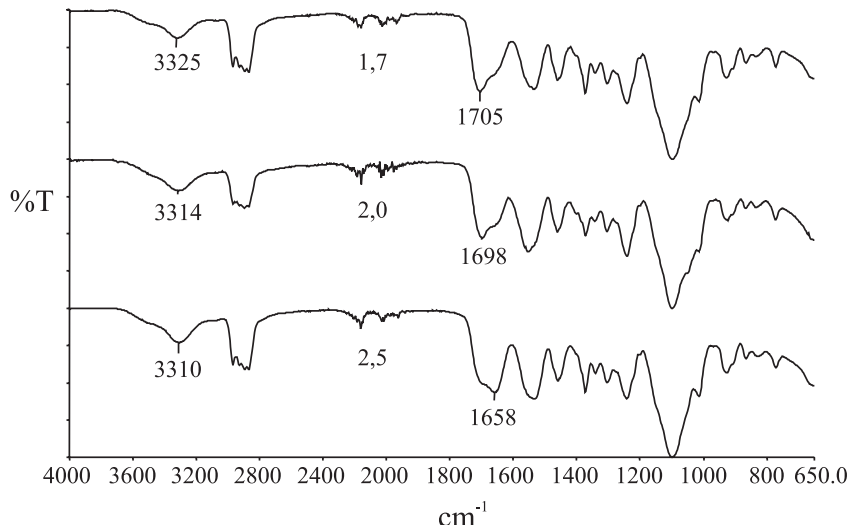

Figura 4. Espectros de absorção dos filmes de PUR da série A com $70 \%$ de $P P G-b-P E G$ e variação na razão $\mathrm{NCO} / \mathrm{OH}$

Os valores dos percentuais máximos de absorção de água, determinados para os filmes de PUR da série A, ficaram compreendidos entre 19 e $80 \%$ e para os filmes da série B, entre 60 e $130 \%$. Isso mostra que os filmes dos PUR formulados apresentam baixa resistência à água. Pode-se atribuir esse comportamento à característica hidrofílica das cadeias, evidenciada na série $\mathrm{B}$, que contém copolímero com maior teor de PEG. A variação na razão $\mathrm{NCO} / \mathrm{OH}$ não mostrou um padrão de comportamento padronizado nas séries estudadas.

\section{Avaliação mecânica}

O comportamento mecânico de elastômeros termoplásticos sob tração é função das interações intermoleculares entre os segmentos rígidos e envolvem o tamanho, a estrutura química e o teor desses segmentos na cadeia. ${ }^{34} \mathrm{~A}$ estrutura e o teor dos segmentos rígidos são parâmetros extremamente importantes e afetam, em grande parte, o comportamento morfológico e o desempenho dos revestimentos dos PUR segmentados. ${ }^{35}$

Enquanto os domínios rígidos conferem resistência mecânica, os segmentos flexíveis, que apresentam características borrachosas, conferem propriedades elastoméricas às cadeias uretânicas ${ }^{1}$. Estudos revelaram que, ao variar a estrutura química dos polióis, ocorrem mudanças na compatibilidade entre os domínios flexíveis e rígidos afetando dessa forma o desempenho mecânico dos revestimentos de poliuretanos dispersáveis em água. ${ }^{35}$
A Tabela 6 mostra os resultados dos ensaios mecânicos obtidos para os filmes vazados produzidos na série $\mathrm{B}$. De forma geral, pode-se observar que a incorporação de PEG-b-PPG na cadeia poliuretânica provocou uma tendência à redução tanto no alongamento na ruptura quanto da tensão na ruptura. Isso mostra que a presença do copolímero PEG-b-PPG diminuiu a resistência do material, isto é, tornou o material mais frágil, pois diminuiu também a sua elasticidade. $\mathrm{O}$ grupamento lateral metila, no PPG e também no homopolímero PPG, impediria a ordenação dos segmentos flexíveis e aumentaria a separação entre as cadeias dos mesmos, aspectos que contribuiriam para a redução da tensão de ruptura do material. ${ }^{24,36,37}$ Em contrapartida, a incorporação de segmentos PEG, provenientes do copolímero PEGb-PPG promoveria uma maior regularidade da estrutura polimérica. Isso foi realmente verificado com a diminuição no alongamento na ruptura dos filmes testados. No entanto, os valores de tensão na ruptura diminuiram, ou seja, a ordenação promovida pelos segmentos PEG não aumentou a resistência mecânica dos materiais. A incorporação do copolímero, que aumentou a rigidez, na verdade tornou as amostras mais frágeis.

Tabela 6. Resistência à tração de filmes de PUR da série $\mathrm{B}$ obtidos com diferentes razões $\mathrm{NCO} / \mathrm{OH}$ e diferentes proporções de copolímero PEG-b-PPG

\begin{tabular}{lcc}
\hline $\begin{array}{l}\text { Reação } \\
\text { NCO/OH/ \% } \\
\text { PEG-b-PPG }\end{array}$ & $\begin{array}{c}\text { Propriedades Mecânicas } \\
\text { Alongamento na } \\
\text { Ruptura }(\%)\end{array}$ & $\begin{array}{c}\text { Tensão na } \\
\text { Ruptura (MPa) }\end{array}$ \\
\hline $1,7 / 00$ & 217 & 2 \\
$1,7 / 05$ & 115 & 3 \\
$1,7 / 10^{\mathrm{a}}$ & 83 & 2 \\
$1,7 / 15^{\mathrm{a}}$ & 46 & 2 \\
$1,7 / 20$ & $\mathrm{~B}$ & $\mathrm{~B}$ \\
$2,0 / 00$ & 76 & 6 \\
$2,0 / 10$ & 45 & 5 \\
$2,0 / 20^{\mathrm{a}}$ & 54 & 3 \\
$2,0 / 30^{\mathrm{a}}$ & 93 & 2 \\
$2,5 / 00$ & $\mathrm{ND}$ & $\mathrm{ND}$ \\
$2,5 / 10$ & $\mathrm{ND}$ & ND \\
$2,5 / 20$ & $\mathrm{ND}$ & ND \\
$2,5 / 30$ & 22 & 9 \\
\hline
\end{tabular}

a. Filme proveniente de dispersão instável frente à sedimentação; B dispersão muito viscosa não permitindo formação de filme; ND - não determinado, filme muito quebradiço.

No entanto, pode-se verificar que, ao se aumentar o teor de ligações uretânicas e ureicas, na razão $\mathrm{NCO} / \mathrm{OH}=2,0 \mathrm{e}$, principalmente, na razão $\mathrm{NCO} / \mathrm{OH}=2,5$, ocorre um aumento significativo na resistência mecânica, provavelmente devido ao aumento das interações por ligações de hidrogênio. . $^{1,7,34}$

É interessante notar que o único filme inteiriço produzido com a razão $\mathrm{NCO} / \mathrm{OH}=2,5$ foi justamente aquele com maior incorporação de segmentos PEG, que seria a amostra com maior rigidez de todas as sintetizadas. Poder-se-ia esperar que essa rigidez tornasse a amostra mais frágil. No entanto, foi ela a que apresentou a maior resistência mecânica. Esse fato inesperado mostra que nessa formulação, os segmentos promotores de rigidez (PEG, grupos uretano e uréia) atuaram sinergicamente formando um material resistente.

\section{CONCLUSÃO}

Sistemas não-poluentes à base de dispersões aquosas de poliuretanos com grupamentos aniônicos e segmentos hidrofílicos foram 
obtidos com teores de sólidos próximos a $40 \%$ e estabilidade para estocagem, variando de acordo com a formulação. A faixa de viscosidade das dispersões foi adequada para aplicações desses sistemas como revestimentos ou adesivos com afinidade por substratos como vidro, alumínio, madeira, papel e cerâmica. De forma geral, as dispersões poliuretânicas mais estáveis e com teor de sólidos mais elevados foram aquelas obtidas com percentuais mais baixos do copolímero em bloco à base de glicol etilênico e glicol propilênico (PEG-b-PPG). A presença de maiores teores de segmentos hidrofílicos PEG, provenientes do copolímero, bem como de maiores proporções de segmentos rígidos, formados por ligações uretânicas e ureicas, elevou o tamanho médio de partículas das dispersões. A incorporação do PEG-b-PPG diminuiu a resistência mecânica e a elasticidade dos poliuretanos, como também, aumentou a afinidade das cadeias poliméricas pela água, provocando aumento na viscosidade das dispersões.

\section{AGRADECIMENTOS}

À CAPES, ao CNPq e à FAPERJ, pelo suporte financeiro e à Dow Química, à Petroflex, à Resinac, à Rio Polímeros e ao CTA/ IAE pela doação de reagentes.

\section{REFERENCIAS}

1. Coutinho, F. M. B.; Delpech, M. C.; Garcia, M. E. F.; Polím.: Ciên. Tecnol. 2004, 14, 234.

2. Delpech, M. C.; Coutinho, F. M. B.; Moura, P. M. Q.; Mello, S. D. S.; Polím.: Ciên. Tecnol. 1996, 3, 30.

3. Mequanint, K.; Sanderson, R.; Eur. Polym. J. 2006, 42, 1145.

4. Coutinho, F. M. B.; Delpech, M. C.; Alves, L. S.; Polím.: Ciên. Tecnol. 2000, 10, 49 .

5. Coutinho, F. M. B.; Delpech, M. C.; Polím.: Ciên. Tecnol. 1999, 9, 41.

6. Coutinho, F. M. B.; Delpech, M. C.; Alves T. L.; Gomes, A. S.; Polím.: Ciên. Tecnol. 2002, 12, 248.

7. Delpech, M. C.; Coutinho, F. M. B.; Polym. Test. 2000, 19, 939.

8. Vega-baudrit, J.; Sibaja-ballestero, M.; Vázquez, P.; Torregrosa-maciá, R.; Martín-Martinez, J. M.; Int. J. Adhes. Adhes. 2007, 27, 469.

9. Król, P.; Prog. Mat. Sci. 2007, 52, 915.

10. Bao, L.; Lan, Y.; Zhang, S.; J. Polym. Res. 2006, 13, 507.

11. Yilgor, I.; Yilgor, E.; Guler, I. G.; Ward, T. C.; Wilkes, G. L.; Polymer 2006, 47, 4105.

12. Sebenik, U.; Krajnc, M.; Int. J. Adhes. Adhes. 2006, 27, 527.
13. Huang, S. L.; Chao, M. S.; Lai, J. Y.; Eur. Polym. J. 1997, 34, 449.

14. Mohaghegh, S. M.; Barikani, M.; Entezami, A. A.; Colloids Surf., A 2006, 276, 95.

15. Subramani, S.; Cheong, I. W.; Kim, J. H. S.; Eur. Polym. J. 2004, 40, 2745.

16. Lee, H.; Wu, S.; Jeng, R.; Colloids Surf., A 2006, 276, 176.

17. Magalhães, L. C.; Coutinho, F. M. B.; Delpech, M. C.; Polím.: Ciên. Tecnol. 2007, 17, 212.

18. Book of American Society for Testing and Materials Standards-ASTM, D 2834-72, Easton, 1975.

19. Hepburn, C.; Polyurethane elastomers, $2^{\text {nd }}$ ed., Elsevier Science Publishers Ltda: New York, 1991, cap. 1.

20. Book of American Society for Testing and Materials Standards - ASTM, D 570-63, Easton, 1979.

21. Book of American Society for Testing and Materials Standards-ASTM, D 638-72, Easton, 1979.

22. Coutinho, F. M. B.; Delpech, M. C.; Alves, L. S.; J. Appl. Polym. Sci. 2001, 80,566

23. Ebrahimi, M. N.; Barikani, M.; Mohaghegh, S. M. S.; Iranian Polym. J. 2006, 15, 323

24. Coutinho, F. M. B.; Alves, L. S.; Delpech, M. C.; Anais Assoc. Bras. Quím.1998, 47, 255.

25. Coutinho, F. M. B.; Delpech, M. C., Moura, P. M. Q.; Mello, S. D. S.; Polym. Bull. 1996, 37, 1.

26. Nanda, A. K.; Wicks, D. A.; Madbouly, S. A.; Otaigbe, J. U.; J. Appl. Polym. Sci. 2005, 98, 2514.

27. Ayres, E.; Oréfice, R. L.; Mansur, H. S.; Anais do $8^{\circ}$ Congresso Brasileiro de Polímeros, São Paulo, Brasil, 2005.

28. Mattos, E. C.; Dutra, R. C. L.; Diniz, M. F.; Iha, K.; Polím.: Ciên. Tecnol. 2004, 14, 63.

29. Subramani, S.; Park, Y.; Lee, Y.; Kim, J.; J. Prog. Org. Coat. 2003, 48, 71.

30. Jhon, K.; In-woo, C.; Kim, J.; Colloids Surf., A 2001, 179, 71.

31. Yen, M.; Tsai, P.; Hong, P.; Colloids Surf., A 2006, 279, 1.

32. http://www.functionalpolymers.basf.com/portal/streamer? find=291079, acessada em Agosto 2006.

33. Kim, B. K; Lee, Y. M.; J. Appl. Polym. Sci. 1994, 54, 1809.

34. Coutinho, F. M. B.; Delpech, M. C.; Polym. Test. 1996, 15, 103.

35. Chattopadhyay, D. K.; Raju, K. V. S. N.; Prog. Polym. Sci. 2007, 43, 1825.

36. Kim, B. K.; Lee, J. C.; Lee, K. H.; J. Macromol. Sci., Part A: Pure Appl. Chem. 1994, A31, 1241.

37. Kim, B. K.; Kim, T. K.; J. Appl. Polym. Sci. 1991, 43, 2295 